\title{
Quantitative Imaging of Specific Proteins in the Human Retina by LA-ICP-MS using Bioconjugated Metal Nanoclusters as Labels
}

\author{
María Cruz-Alonso ${ }^{1}$, Beatriz Fernandez**1,3 ${ }^{\text {, Montserrat García-Díaz }}{ }^{2,3}$, Héctor \\ González-Iglesias $^{* 2,3}$ and Rosario Pereiro ${ }^{1,3}$
}

${ }^{1}$ Department of Physical and Analytical Chemistry, University of Oviedo, Julian Claveria 8, 33006 Oviedo, Spain.

${ }^{2}$ Instituto Oftalmológico Fernández-Vega, Avda. Dres. Fernández-Vega, 34, 33012 Oviedo, Spain.

${ }^{3}$ Instituto Universitario Fernández-Vega (Fundación de Investigación Oftalmológica, Universidad de Oviedo), Spain.

*Authors to whom correspondence should be addressed:

fernandezbeatriz@uniovi.es, hgonzalez@ fio.as 


\begin{abstract}
A sensitive methodology using antibody-conjugated gold nanoclusters (AuNCs) was developed for the quantitative bioimaging of specific proteins in biological tissues by laser ablation (LA) coupled to inductively coupled plasma - mass spectrometry (ICP-MS). Determination of metallothioneins (MT1/2 protein isoforms) images in human retina tissue sections was carried out as a proof of concept. AuNCs used as label were conjugated to the selected antibody through carbodiimide coupling. A stoichiometry "AuNCs:available antibody" of 1:1 was obtained. The high amplification provided by AuNCs labels allowed obtaining the distribution of MT1/2 in the neurosensory retina layers ( $5 \mu \mathrm{m}$ thick sections) by LA-ICP-MS. Elemental images of ${ }^{197} \mathrm{Au}^{+}$were quantified with gelatin matrix-matched standards and then converted to 2D quantitative images of MT1/2 concentration. For validation purposes, average concentrations of MT1/2 obtained in the human retinal layers by LA-ICP-MS were successfully compared with those obtained with a commercial ELISA kit.
\end{abstract}

KEYWORDS: Gold nanoclusters; Immunohistochemical analysis; Proteins; Quantitative bioimaging; Laser ablation ICP-MS. 


\section{INTRODUCTION}

Laser ablation - inductively coupled plasma - mass spectrometry (LA-ICP-MS) offers interesting capabilities for elemental and isotopic direct solid analysis, including high spatial resolution (in the low $\mu \mathrm{m}$ range) and sensitivity $\left(\mu \mathrm{g} \mathrm{g}^{-1}-\mathrm{ng} \mathrm{g}^{-1}\right)$. Furthermore, LA-ICP-MS has shown a huge potential for elemental imaging studies in biological tissues. ${ }^{1,2}$ Moreover, by using elemental-labeled antibodies (Ab) in combination with LA-ICP-MS, the imaging of specific proteins can also be achieved. ${ }^{3}$

Single metal chelates coordinated with heteroatoms ${ }^{3}$ and polymeric tags containing several metal chelates (such as the $\mathrm{MaxPar}^{\mathrm{TM}}$ commercial kit) ${ }^{4}$ have been successfully employed for elemental labeling of antibodies for protein LA-ICP-MS imaging. An important advantage of polymeric tags compared to single metal chelates is their higher signal amplification. These polymers have been used in varied applications mostly in combination with ICP-TOFMS for mass cytometry, ${ }^{5}$ where they have shown an outstanding value. In addition, the strategy has been coupled to LA for imaging mass cytometry $^{6}$ and for highly multiplexed imaging of tissues at subcellular resolution. ${ }^{4}$ Unfortunately, polymeric labels are composed of a non-metallic part and, therefore, they have a relatively large size, which can hinder an efficient Ab-antigen interaction. Moreover, it has been reported that they show risk of non-specific interactions with the sample for the analysis of proteins in tissue sections. ${ }^{7}$

The use of small metal nanoclusters $(\mathrm{NCs}),{ }^{8}$ is other alternative for specific protein imaging by LA-ICP-MS. The number of metal atoms per NC is higher compared to a polymeric tag and greater amplification can be achieved with a smaller size (each NC, with typical diameters of 2-3 nm, has $\sim 500$ metal atoms). ${ }^{9}$ Moreover, these metal NCs can be fluorescent. ${ }^{8}$ Therefore, they offer possibilities for multimodal imaging (through fluorescence and MS analysis) in the same tissue section. In a previous work we demonstrated such multimodal imaging potential of AuNCs in the study of proteins distribution in ocular tissues: high resolution qualitative images were successfully obtained for Au distribution along the retina region. ${ }^{10}$

Concerning protein analyses, many methods only provide protein-level variations between two samples (differential expression proteomics). For example, conventional methods for protein imaging in biological tissues, such as immunoperoxidase staining and immunohistofluorescence, allow obtaining semi-quantitative information by computer-assisted image analysis. ${ }^{11}$ However, absolute quantitative information is required to have a better understanding of the biological mechanisms and to know 
possible biological implications of biomolecules. Therefore, a further challenge for protein imaging studies in biological tissues is related to the opportunity offered by MSbased techniques to obtain absolute quantitative information through elemental-labels. ${ }^{12}$

Quantitative elemental information of heteroatoms naturally present in biological tissues can be currently obtained using LA-ICP-MS by resorting to appropriate calibration strategies (e.g. homogenized and spiked tissues, gelatin standards, agarose gel standards and polymeric films). ${ }^{2,13,14}$ However, specific protein quantification of images in biological tissues is a more challenging task. Studies on quantitative imaging of biomolecules in tissue sections by LA-ICP-MS are scarce. Mueller et al. ${ }^{15}$ presented an interesting approach, using lanthanide-labelled antibodies (MAXPAR ${ }^{\mathrm{TM}}$ ) for quantitative immuno-imaging of single eukaryotic cells. The calculation of an exact labelling degree as well as the correlation factor between amount of lanthanide per cell and amount of antibody-antigen complex were reported as critical aspects for further optimization.

The aim of our investigation is the development of a method for absolute quantitative imaging of specific proteins in tissue sections by LA-ICP-MS. As a proof of concept, an immunohistochemical procedure based on the use of AuNCs bioconjugated with the selected Ab via carbodiimide crosslinking (we focused on carbodiimide chemistry because it is the most prevalent conjugation strategy used in nanotechnology today) is presented for the specific localization of metallothioneins 1 and $2($ MT1/2) in human retina sections. MTs are a family of proteins exhibiting diverse functions, including transport and storage of essential metal ions, detoxification of metals, neuroprotection and defense against oxidative damage. ${ }^{16,17}$ In particular, MTs are highly expressed in the human eye and have been suggested to play a key role in the protection of neuronal retinal cells against oxidative stress. ${ }^{17,18}$ The retina is composed by different layers of neuronal cells, where the expression profile of MTs is different between layers. Thus, it is of great interest to obtain quantitative information distribution of MT1/2 in the retina as a proof of concept.

To corroborate the quantitative protein content of MT1/2 obtained by LA-ICP-MS in the human retina, a commercial ELISA kit was employed with dissected human retinas from post mortem donors. 


\section{EXPERIMENTAL SECTION}

Chemicals and Materials. All solutions were prepared with distilled deionized water obtained from Milli-Q water purification system (Millipore). $\mathrm{NaAuCl}_{4} \cdot \mathrm{H}_{2} \mathrm{O}(99 \%$ powder; Sigma-Aldrich) as metal precursor, lipoic acid (> 98\% powder; Across Organics) as surface ligand and $\mathrm{NaBH}_{4}$ (> 98\% powder, Sigma-Aldrich) as reducing agent were employed for the synthesis of AuNCs.

Immunoassays in the human eye tissue sections were performed with a primary monoclonal anti-MT1/2 Ab produced in rabbit (Abcam). 1-Ethyl-3-(3dimethylaminopropyl) carbodiimide (EDC) (98\% powder; Across Organics) and Nhydroxysuccinimide (NHS) (95\% powder; Thermo Fisher Scientific) were used in the bioconjugation procedure. To reduce non-specific interactions, hydroxylamine hydrochloride ( $\geq 99 \%$ powder; Sigma-Aldrich) was used at the last step of the $\mathrm{Ab}$ labelling procedure. Amicon ultra centrifugal filter units (10 and $100 \mathrm{kDa}$ pore size, Millipore) were used for purification stages.

Triton X-100 (Sigma-Aldrich) was used to permeabilize tissue sections and bovine serum albumin (BSA) (99\% powder, Sigma-Aldrich) and goat serum (SigmaAldrich) were employed as blocking agents in immunoassay procedures. Washing steps in the immunoassay were done with phosphate buffered saline solution (PBS, $10 \mathrm{mM}$, $\mathrm{pH}$ 7.4) (Sigma-Aldrich). For the calibration by LA-ICP-MS, standards of gelatin (Merck) were used. Finally, human Zn-MT ELISA kit (MyBioSource) was used to quantify the MT1/2 levels in dissected human retinas from post mortem donors.

Instrumentation. LA-ICP-MS analyses were carried out using a commercial LA system (LSX-213 from Teledyne Cetac Technologies) coupled to a double-focusing sector field ICP-MS (Element 2, Thermo Fisher Scientific) working at low mass resolution mode. The commercial ablation cell was replaced by a novel Peltier-cooled ablation cell built in-house with a reduced internal volume that allows additionally keeping cell temperature constant at $-20{ }^{\circ} \mathrm{C}$. Experimental parameters used for LA-ICPMS analysis are collected in Supporting Information (Table S1).

Synthesis and characterization of anti-human MT1/2-AuNCs. AuNCs were synthesized following the procedure described elsewhere. ${ }^{19,20}$ AuNCs with a diameter particle size of $2.7 \pm 0.1 \mathrm{~nm}$ were obtained $(\mathrm{n}=500,99 \%$ confidence intervals obtained 
with the standard normal distribution), with an average of 579 atoms of Au per NC. ${ }^{20}$ The $\mathrm{Ab}$ labelling procedure was performed following the protocol previously described. ${ }^{10} \mathrm{~A}$ 1:3 molar ratio of Ab:AuNCs in the labelling solution was found as the optimum value. Additionally, such anti-human MT1/2-AuNCs bioconjugate was incubated with hydroxylamine hydrochloride to reduce further nonspecific interactions in the biological tissues.

To determine the available $\mathrm{Ab}$ concentration in the Ab-AuNCs, an ELISA was performed (see Supplementary Information) with a mouse IgG protein standard produced with a known concentration in "The Biotechnology Preparative" Unit of the Scientific-Technical Services of the University of Oviedo. Moreover, to characterize the Ab-AuNCs stoichiometry, sodium dodecyl sulfate-polyacrylamide gel electrophoresis (SDS-PAGE) was employed using denaturing 1D-PAGE. Experimental procedures and gel conditions used (Table S2) are collected in Supporting Information.

Collection of human eyes from post mortem donors. For imaging analysis, human eye globes from adult donors without any signs of relevant ocular pathologies were obtained $24 \mathrm{~h}$ post mortem through the National Disease Research Interchange (Philadelphia, USA). The samples were formalin fixed and paraffin embedded (FFPE) following conventional protocols. The procedures adhered to the tenets of the Declaration of Helsinki. LA-ICP-MS analyses were performed with eye tissue sections of 5- $\mu \mathrm{m}$ thickness from FFPE blocks that were placed in FLEX coated microscope slides (IHC Microscope slides, Agilent Technologies). Deparaffinization procedure was done with xylene and ethanol (Sigma-Aldrich).

For MT1/2 quantification in human retinas by commercial ELISA, a total of 8 eye globes from adult human donors without relevant ocular pathologies affecting the retina, ranging in age from 45 to 75 years old, were obtained $24 \mathrm{~h}$ post mortem through the Hospital Universitario Central de Asturias (HUCA, Oviedo, Spain). Each eye was dissected and the retina separately collected and stored at $-80{ }^{\circ} \mathrm{C}$ until further use. All the procedures adhered to the tenets of the Declaration of Helsinki, and full ethical approval was obtained from the Clinical Research Ethics Committee at the HUCA.

Immunoassay protocol for LA-ICP-MS analysis. The tissue sections mounted on microscope slides were immersed in xylol for paraffin removal and, finally, they were rehydrated in a decreasing graded alcohol series of ethanol $(100 \%, 95 \%, 80 \%$ and 
$70 \%$ ) for 5 min per condition. Afterwards, tissue sections were washed three times (10 min) with $10 \mathrm{mM}$ PBS pH 7.4 and, subsequently, incubated with $0.1 \%$ triton $\mathrm{X}-100$ in PBS (5 min). Next, another sequence of washing steps with PBS (10 min, three times) was performed and the slides were then incubated with the blocking agent solution (0.1\% BSA and $10 \%$ goat serum solution in $10 \mathrm{mM} \mathrm{PBS,} \mathrm{pH} 7.4)$ at room temperature for $30 \mathrm{~min}$. Subsequently, the Ab-AuNC bioconjugate $\left(10 \mu \mathrm{g} \mathrm{mL} \mathrm{m}^{-1}\right)$ was added and incubated overnight at $4^{\circ} \mathrm{C}$. After this incubation, a final washing step with PBS was done and the slides were stored at $-20{ }^{\circ} \mathrm{C}$ until LA-ICP-MS analysis.

Preparation of gelatin standards for quantitative LA-ICP-MS analyses. Quantitative distribution of $\mathrm{Au}$ in sections from human eyes was carried out by LAICP-MS using laboratory gelatin standards. Preparation of such matrix-matched standards is collected in Supporting Information.

Determination of MT1/2 concentration in the human retina by ELISA. Quantification of MT1/2 by ELISA was carried out in the water-soluble protein fraction (i.e., cytosol) of previously dissected retinas. To this end, collected retinas were weighed and homogenized in PBS (100 $\mu \mathrm{L}$ per $10 \mathrm{mg}$ of tissue) and the resulting suspension was subjected to ultrasonication (three 30 -s pulses at $4{ }^{\circ} \mathrm{C}$ using the probe Sonoplus HD 2070 Bandelin) to break the cell membranes. Finally, the homogenates were centrifuged for $15 \mathrm{~min}$ at $1500 \times \mathrm{g}$, and the supernatants (cytosolic fraction of retinal cells) were collected and stored at $-80{ }^{\circ} \mathrm{C}$ until ELISA quantification, following manufacturer procedures.

\section{RESULTS AND DISCUSSION}

Characterization of the Ab-AuNCs bioconjugate. In order to use the $\mathrm{Ab}$ AuNCs for quantification of MT1/2 protein in the human retina, it is necessary to know its stoichiometry (i.e. the number of AuNCs per Ab molecule). As reported by Song and

Chan, ${ }^{21}$ the number $(\mathrm{N})$ of AuNCs bound to an $\mathrm{Ab}$ can be determined following Equation [1] as the ratio between the $\mathrm{Ab}$ concentration $\left(\mathrm{C}_{\mathrm{Ab}}\right)$ and the concentration of AuNCs $\left(\mathrm{C}_{\mathrm{AuNCs}}\right)$ in the purified bioconjugate. 


$$
N=\frac{C(A u N C s)}{C(A b)} \quad \text { Equation [1] }
$$

It is important to note that the determination of total protein per bioconjugate unit will not provide enough information because some Ab could be partially destroyed or impeded for interaction with the analyte. Therefore, to measure the concentration of $\mathrm{Ab}$ available for reaction with the corresponding protein, a direct ELISA test was performed. In our experiments, the purified bioconjugate (Ab-AuNCs) and the $\mathrm{Ab}$ without AuNCs were subjected to a quantitative ELISA with an IgG protein standard as described in the Experimental section. An $\operatorname{IgG}$ protein was incubated at different concentrations to perform the calibration curve. Figure S1 (Supporting Information) shows the calibration curve obtained by ELISA using the mouse IgG protein for the quantification of $\mathrm{Ab}$ and $\mathrm{Ab}$-AuNCs. Experimental results showed that $\mathrm{Ab}$ concentration for the purified bioconjugate (Ab-AuNCs) and the no conjugated $\mathrm{Ab}$ was $5.47 \pm 0.23 \mu \mathrm{g} \mathrm{mL}^{-1}$ and $5.78 \pm 0.19 \mu \mathrm{g} \mathrm{mL}^{-1}$, respectively (uncertainties represent the standard deviations of the mean of 3 independent measurements). The obtained $\mathrm{Ab}$ concentrations demonstrate the high Ab bioconjugation yield (95\%) with the proposed methodology. Then, considering the $\mathrm{Ab}$ concentration, its molecular mass (approximately $150 \mathrm{kDa}$ ) and the Avogadro constant, it is possible to obtain the $\mathrm{Ab}$ concentration in terms of number of $\mathrm{Ab}$ per $\mathrm{mL}$ : an average value of $2.20 \cdot 10^{16}$ molecules of $\mathrm{Ab} \mathrm{mL} \mathrm{L}^{-1}$ was found in the purified $\mathrm{Ab}$-AuNCs bioconjugate solution.

In a second step, the AuNCs concentration in the Ab-AuNCs was determined by conventional nebulization and ICP-MS detection. For this purpose, the concentration of $\mathrm{Au}$ was determined in the Ab-AuNCs purified solution. Au calibration (in the range of 0-20 $\mathrm{ng} \mathrm{mL}^{-1}$ ) was performed after the acidic digestion of the samples with a mixture of $\mathrm{HNO}_{3}: \mathrm{HCl}\left(1: 3\right.$ molar ratio). Taking into account that $1 \mathrm{NC}$ contains $9.62 \cdot 10^{-22}$ moles of $\mathrm{Au},{ }^{20}$ the AuNCs concentration in the purified Ab-AuNCs bioconjugate was found to be $2.17 \cdot 10^{16}$ molecules of AuNCs $\mathrm{mL}^{-1}$. According to Equation [1], the number of AuNCs bound per Ab molecule is approximately 0.99. Therefore, the obtained average value for $\mathrm{N}$ allows us to establish that experimental stoichiometry of Ab:AuNCs bioconjugate is $1: 1$.

To further characterize the bioconjugate and confirm the number of labels per $\mathrm{Ab}$, SDS-PAGE was subsequently employed. The experimental electrophoresis conditions are collected in Table S2. The Ab-AuNCs bioconjugate, the Ab alone, and the AuNCs 
alone were submitted to gel electrophoretic separation using independent lanes. Figure 1 shows the electrophoretic profile obtained for the molecular mass marker (lane A) and the different samples. As expected, no colorimetric bands were identified for the AuNCs (lane B in Figure 1) due to Coomassie stain only blinds to proteins. However, fluorescence signal from AuNCs was measured directly in the gels by confocal microscopy (data not shown). Due to their low particle size, AuNCs fluorescence was easily identified at the dye front (at the bottom of the gel). Concerning the bands from $\mathrm{Ab}$-AuNCs and Ab, a slight shift between the bands obtained for the Ab-AuNCs bioconjugate and the $\mathrm{Ab}$ was observed in the gel (lanes $\mathrm{C}$ and $\mathrm{D}$, respectively). Such shift is more pronounced in the case of the heavy chain of the Ab compared to the light chains but can be observed such displacement for the two bands.

To quantify the shift observed for the bands of the $\mathrm{Ab}$ and the Ab-AuNCs, determination of the molecular mass in the different bands of the gels was performed. Molecular masses can be determined by comparing the mobility of assayed test proteins (Ab and Ab-AuNCs) to the mobility of known protein markers. For such purpose, the relative migration distance of the individual protein bands was measured relatively to the mobility of the ion front. ${ }^{22}$ In practice, relative mobility is calculated as the quotient of the distance travelled by a protein from the top of the resolving gel divided by the distance migrated by the ion front. Taking into account the different bands observed in the gel for the molecular mass size marker and the relative mobility, a semilogarithmical scaled calibration curve was obtained. Figure S2 shows the calibration curve obtained for the molecular mass versus the relative mobility. Additionally, Table 1 collects the results obtained for the molecular mass of each chain in the Ab (with and without AuNCs as labels) determined by their relative mobility in the gel (using as mobility value the middle of each band). Experimental results showed a slight difference in the molecular mass obtained for the light chain by comparing $\mathrm{Ab}$ and $\mathrm{Ab}$ AuNCs. However, a more significant difference was observed for the heavy chain $(62.36 \pm 5.9 \mathrm{kDa}$ and $72.62 \pm 7.3 \mathrm{kDa}$, respectively). Such molecular mass difference was about $10.3 \mathrm{kDa}$, which corresponds with the molecular mass of one AuNC. ${ }^{23}$ Thus, it can be confirmed that stoichiometry of $\mathrm{Ab}$-AuNCs corresponds to one $\mathrm{AuNC}$ per $\mathrm{Ab}$.

Calibration by LA-ICP-MS with matrix-matched standards. In the present study, the quantitative imaging of MT1/2 in the human retina region was pursued. For such purpose, external calibration based on Au standard-spiked gelatin sections ( $5 \mu \mathrm{m}$ 
thick) was proposed. The use of synthetic spiked gelatin standards is of growing interest because their density and water content simulates biological tissues. Furthermore, they have been successfully employed for quantification of heteroatoms in different biological tissues. ${ }^{13,14}$ Concerning internal normalization for quantitative imaging analyses, different strategies have been proposed using LA-ICP-MS. ${ }^{24,25}$ In the present work, it is ensured that ablated mass between sample and standards was comparable because the entire thin sections were ablated in both cases. ${ }^{26}$ Moreover, correction by density differences between gelatin and the retina tissue was also carried out: experimental value obtained for the density of the gelatin was $1.14 \mathrm{~g} \mathrm{~cm}^{-3}$ whereas the retina exhibited an average density value of $1.02 \mathrm{~g} \mathrm{~cm}^{-3}$. Correction of possible sensitivity drift was achieved by measuring the gelatin standard before and after each sample imaging (standard-sample bracketing calibration).

In this work, two different calibrations by LA-ICP-MS were explored using gelatin standards spiked either with the $\mathrm{Au}$ salt precursor (i.e. $\mathrm{NaAuCl}_{4} \cdot \mathrm{H}_{2} \mathrm{O}$ ) or with the synthetized AuNCs used in the bioconjugation. This study was done to check if there are significant differences in the $\mathrm{Au}$ signal depending on the $\mathrm{Au}$ standard employed (i.e. in salt form or as a metal NC). Also, potential risk of heterogeneity problems in the case of colloidal gold (AuNCs) was tested. Figure S3 (Supporting Information) shows the ${ }^{197} \mathrm{Au}^{+}$signal (in logarithmic scale) obtained by LA-ICP-MS for the analysis of the gelatin standards, using $\mathrm{NaAuCl}_{4} \cdot \mathrm{H}_{2} \mathrm{O}$ (Figure S3A) and AuNCs (Figure S3B). A stable signal was observed for the gelatin with AuNCs indicating a homogeneous distribution. Moreover, no significant differences in terms of sensitivity were observed by comparison of ${ }^{197} \mathrm{Au}^{+}$profiles, which can be attributed to the small size of our AuNCs (2-3 nm) and to the laser ablation process compared to conventional nebulization ICP-MS. Therefore, for the sake of simplicity the Au salt precursor was used to spike the gelatin standards, with a final concentration ranging from 0 to $60 \mu \mathrm{g} \mathrm{g}$ 1. Figure S4 (Supporting Information) shows the calibration curve constructed by plotting the signal intensity of ${ }^{197} \mathrm{Au}^{+}$obtained by LA-ICP-MS versus the Au-standards concentration. The correlation coefficient was 0.999 .

Additionally, the limit of detection (LOD) for Au was calculated by using the $3 \mathrm{~s}$ criterion $\left(3 \mathrm{~s}_{\mathrm{b}} / \mathrm{S}\right)$, where $\mathrm{s}_{\mathrm{b}}$ is the standard deviation of 5 independent blank signals (i.e. gelatin standard without $\mathrm{Au}$ ) and $\mathrm{S}$ is the $\mathrm{Au}$ sensitivity obtained in the calibration graph. The LOD for Au was found to be $8.3 \mathrm{ng} \mathrm{g}^{-1}$. 
Quantitative imaging of MT1/2 in the human retina. The proposed quantitative imaging methodology based on the use of AuNCs as Ab label was applied to study the MT1/2 distribution in neural retina tissue sections from post mortem donors. Human eye tissue sections following immunohistochemistry were ablated in scanning mode $(10 \mu \mathrm{m}$ laser spot diameter) by LA-ICP-MS. Using the obtained linear regression with the gelatin standards (Figure S4), each data point (or pixel) resulting from the qualitative images was converted from net intensities into concentrations (Au concentration at a

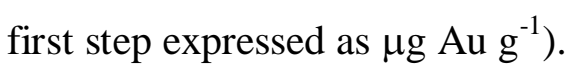

As the final goal is to obtain quantitative images of MT1/2 (expressed as $\mu \mathrm{g}$ of MT per $g$ of tissue), different calculations should be done. Figure 2 illustrates a flowdiagram with the different steps required to convert detected Au concentration into sought MT1/2 concentration. In a previous work, it was found that in one AuNC there are $9.62 \cdot 10^{-22}$ moles of $\mathrm{Au}^{20}$ Thus, after LA-ICP-MS measurements of ${ }^{197} \mathrm{Au}^{+}$it was obtained the concentration of $\mathrm{Au}$ (expressed as moles $\mathrm{g}^{-1}$ ) in each pixel, and so the corresponding concentration of AuNCs $\left(\mathrm{mol} \mathrm{g}^{-1}\right)$ per sample pixel. As stated above, in the Ab-AuNC characterization it was determined that the stoichiometry of the bioconjugate corresponds to one AuNC per $\mathrm{Ab}$. This allows us to know the $\mathrm{Ab}$ concentration (moles $\mathrm{g}^{-1}$ ) in each sample pixel of the tissue.

On the other hand, interaction between the $\mathrm{Ab}$ and the sought protein in the biological tissue has to be quantitative. According to this, three main points should be met: to use a thin sample in order to ensure full bioconjugate penetration in the sample tissue, to employ an $\mathrm{Ab}$ with a high affinity constant, ${ }^{21}$ and to incubate the tissue section with a high excess of bioconjugate (also it is important an efficient removal of unreacted bioconjugate). Since in our experiments all the above conditions were met, a recognition stoichiometry 1:1 (Ab-antigen) was assumed. This allowed the calculation of the analyte concentration in $\mathrm{mol} \mathrm{g}^{-1}$ (MT1/2 in this particular case) per sample pixel. Finally, by using the molecular mass of MT1/2 ( 6.5 kDa), moles of MT1/2 can be converted to $\mu \mathrm{g}$.

Figure 3 shows the quantitative images (expressed in $n g$ MT1/2 $\mathrm{g}^{-1}$ ) obtained for the analysis of the human retina sections from 4 post mortem donors by LA-ICP-MS (Figures 3B, 3C, 3D and 3E, respectively). Tissue sections were subjected to the immunohistochemical protocol proposed using the AuNCs bioconjugated to antiMT1/2. ${ }^{197} \mathrm{Au}^{+}$signal measured by LA-ICP-MS was converted into analyte 
concentration following the steps summarized in Figure 2. Figure 3A represents the transmission image of the ocular tissue analyzed by LA-ICP-MS from donor \#1, specifically the neural retina region consisting of several cell types organized into the distinct marked layers. Figure 3B shows the quantitative image obtained for the same tissue section, being MT1/2 mainly located in the inner nuclear layer, the outer nuclear layer and in the ganglion cells layer. This specific MT1/2 distribution pattern in the human retina was previously reported. ${ }^{10,27}$ Quantitative images obtained for the donors \#2, \#3 and \#4 (Figure 3C, 3D and 3E, respectively) showed similar protein distribution. It can be observed some differences on MT1/2 distribution along the retinal layers attributed to biological diversity characteristics for each individual patient, and to the preferential heterogeneous localization of MTs in the retina.

To determine the average concentration for MT1/2 in the human retina, the arithmetic mean of the protein concentration was calculated for each pixel of the images, showing a MT1/2 concentration that ranges from 64 to $267 \mathrm{ng}$ protein $\mathrm{g}^{-1}$ (267 ng MT1/2 $\mathrm{g}^{-1}$ donor \#1, 126 ng MT1/2 $\mathrm{g}^{-1}$ donor \#2, 64 ng MT1/2 $\mathrm{g}^{-1}$ donor \#3, and 126 ng MT1/2 $\mathrm{g}^{-1}$ donor \#4). Although significant differences were obtained for the maximum protein concentration depending on the sample (e.g. $1500 \mathrm{ng} \mathrm{g}^{-1}$ for donor \#1 and $390 \mathrm{ng} \mathrm{g}^{-1}$ for donor \#4), the average MT1/2 concentration in the retina region are in agreement with the concentration ranges reported for MT1/2 in eye tissues. ${ }^{28-30}$

In order to corroborate the average MT1/2 concentrations obtained by LA-ICPMS using the AuNCs-based immunohistochemistry methodology, the quantification of MT1/2 was performed with a commercial ELISA kit (see Experimental section). Table 2 shows the protein concentrations obtained for the tissue homogenates from 8 different donors, in terms of dry tissue. In this case, MT1/2 concentration ranges from 19 to 268 ng protein $\mathrm{g}^{-1}$, which is the same range than the concentration reported by the LA-ICPMS using the developed methodology. Considering that MTs are cytosolic proteins, we must stress that MT1/2 have been quantified in the water-soluble fraction of neural retina cells. It should be stated that, according to Table 2, the concentrations determined by ELISA show an associated uncertainty value significantly high, due to intrinsic limitations of the ELISA and to the dissection protocol of the retinas. However, this methodology is the most commonly used for quantitative analysis of proteins. In some cases, the two eyes of the same donor were analyzed (denoted as RE and LE in Table 2). Even for the same patient, significant differences in the MT1/2 concentration was found (particularly remarked for donors \#11242 and \#11253), which certainly indicates 
differences in the proper dissection of the retinas. Overall, the results obtained indicate the successful quantification towards the protein imaging in the retina region using $\mathrm{Ab}$ AuNCs bioconjugate as a sensory probe in an immunohistochemistry procedure.

\section{CONCLUSIONS}

Quantitative imaging of specific proteins in biological tissues after immuhistochemical procedures is a goal which was not fully achieved yet. Important advances have been carried out in the development of labels with different emission lines for multiplexed fluorescence analysis. However, difficulty in measuring simultaneously targets (that differ in abundance by an order of magnitude or more), risks of dye fluorescence quenching, sample autofluorescence, signal scattering and lack of robust quantification procedures makes problematic the quantitative imaging by fluorescence measurements.

In this work, the quantitative capabilities of LA-ICP-MS for elemental imaging have been exploited for quantitative imaging of specific proteins in biological tissues. For such purpose, a methodological strategy was developed and results were corroborated by ELISA measurements. In our proof of concept, AuNCs were employed as metal-label of a selected primary $\mathrm{Ab}$, thus allowing for further multiplexing applications using different metal-labels (e.g. different metal NCs) per selected specific Ab. The calculated LOD for the MT1/2 is about $0.5 \mathrm{ng} \mathrm{g}^{-1}$, considering that (i) the LOD for $\mathrm{Au}$ of $8.3 \mathrm{ng} \mathrm{g}^{-1}$ was obtained using the $5 \mu \mathrm{m}$ thick gelatin standards, (ii) each AuNC contains an average of 579 atoms of $\mathrm{Au}$ per NC, and (iii) each detected AuNC corresponds to one MT1/2 molecule (6 kDa).

Finally, it is important to note that the developed quantification strategy is of general application to other antibodies and other metal-labels (e.g. PtNCs and AgNCs), thus opening new avenues for the quantitative imaging of specific proteins in varied biological tissues. Although the knowledge of qualitative proteins distribution in tissue sections can be sometimes enough to provide valuable information, absolute quantification of proteins along tissue structures may provide a more complete understanding of the underlying molecular mechanisms of diseases, where specific biomolecules may have a key role (e.g. Age related macular degeneration, Parkinson disease, Alzheimer, etc.). By using the state-of-the-art instrumentation (e.g. very small laser spots, ultra-fast ablation cells and simultaneous ICP-MS detection systems, such as 
ICP-TOFMS) high resolution images of protein quantitative distribution in biological samples could be obtained in a short analysis time.

\section{Acknowledgements}

This work was supported by project CTQ2016-79015-R by Agencia Estatal de Investigación (Spain) and FEDER. Beatriz Fernandez acknowledges her research contract RYC-2014-14985 to the Spanish Ministry of Economy and Competitiveness through "Ramón y Cajal Program". The Instituto Oftalmológico Fernández-Vega and Fundación de Investigación Oftalmológica acknowledge financial support from “Cátedra Rafael del Pino" (Fundación Rafael del Pino), and "Instituto de Desarrollo Económico del Principado de Asturias" and FEDER (project IDE/2016/000214). The authors show their gratitude to the "The Biotechnology Preparative" unit of the Scientific-Technical Services of the University of Oviedo for assistance in the immunoassays. 


\section{REFERENCES}

(1) Sussulini, A.; Becker, J. S.; Becker, J. S. Mass Spectrom. Rev. 2017, 36, 47-57.

(2) Pozebon, D.; Scheffler, G. L.; Dressler, V. L. J. Anal. At. Spectrom. 2017, 32, 890919.

(3) Giesen, C.; Mairinger, T.; Khoury, L.; Waentig, L.; Jakubowski, N.; Panne, U. Anal. Chem. 2011, 83, 8177-8183.

(4) Giesen, C.; Wang, H. A. O.; Schapiro, D.; Zivanovic, N.; Jacobs, A.; Hattendorf, B.; Schüffler, P. J.; Grolimund, D.; Buhmann, J. M.; Brandt, S.; Varga, Z.; Wild, P. J.; Günther, D.; Bodenmiller, B. Nature Methods 2014, 11, 417-422.

(5) Tanner, S. D.; Baranov, V. I.; Ornatsky, O. I.; Bandura, D. R.; George, T. C. Cancer Immunol- Immunother: CII. 2013, 62, 955-965.

(6) Chang, Q.; Ornatsky, O.I.; Siddiqui, I.; Loboda, A.; Baranov, V. I.; Hedley, D.W. Cytometry Part A 2017, 91A, 160-169.

(7) Waentig, L.; Jakubowski, N.; Hardt, S.; Scheler, C.; Roos, P. H.; Linscheid M. W. J. Anal. At. Spectrom. 2012, 27, 1311-1320.

(8) Zhang, L.; Wang, E. Nano Today 2014, 9, 132-157.

(9) Le Guével, X. IEEE J. Sel. Top. Quant. 2014, 20, 45-56.

(10) Cruz-Alonso, M.; Fernandez, B.; Álvarez L.; González-Iglesias, H.; Traub, H.; Jakubowski, N.; Pereiro, R. Microchim. Acta 2018, 185, 64 (9 pp).

(11) Taylor, C. R.; Levenson, R. M. Histopathology 2006, 49, 411-424.

(12) Calderón-Celis, F.; Encinar, J. R.; Sanz-Medel, A. Mass Spectrom. Rev. 2017, 123.

(13) Limbeck, A.; Galler, P.; Bonta, M.; Bauer, G.; Nischkauer, W.; Vanhaecke, F. Anal. Bioanal. Chem. 2015, 407, 6593-6617.

(14) Rodríguez-Menéndez, S.; Fernández, B.; García, M.; Álvarez, L.; Fernández, M. L.; Sanz-Medel, A.; Coca-Prados, M.; Pereiro, R.; González-Iglesias, H. Talanta 2018, $178,222-230$.

(15) Mueller, L.; Herrmann, A. J.; Techritz, S.; Panne, U.; Jakubowski, N. Anal. Bioanal. Chem. 2017, 409, 3667-3676.

(16) Maret, W.; Krężel A. Mol. Med. 2007, 13, 371-375.

(17) Gonzalez-Iglesias, H.; Alvarez, L.; García, M.; Petrash, C.; Sanz-Medel, A.; CocaPrados, M. Metallomics 2014, 6, 201-208. 
(18) Suemori, S.; Shimazawa, M.; Kawase, K.; Satoh, M.; Nagase, H.; Yamamoto, T.; Hara, H. Invest. Ophthalmol. Visual Sci. 2006, 47, 3975-3982.

(19) Aldeek, F.; Muhammed, M. H.; Palui, G.; Zhan, N.; Mattoussi, H. ACS Nano 2013, 7, 2509-2521.

(20) Cruz-Alonso, M.; Trapiella-Alfonso, L.; Fernández J. M. C.; Pereiro, R.; SanzMedel, A. Biosens. Bioelectron. 2016, 77, 1055-1061.

(21) Song, F.; Chan,W. C. Nanotechnol. 2011, 22, 494006.

(22) Holtzhauer, M. In Basic Methods for the Biochemical Lab. Springer, 2006. ISBN 978-3-540-32786-8.

(23) Erickson, H. P. Biol. Proced. Online 2009, 11, 32-51.

(24) Konz, I.; Fernández, B.; Fernández, M. L.; Pereiro, R.; González-Iglesias, H.; Coca-Prados, M.; Sanz-Medel, A. Anal. Bioanal. Chem. 2014, 406, 2343-2348.

(25) Frick, D. A.; Giesen, G.; Hemmerle, T.; Bodenmiller, B.; Günther, D. J. Anal. At. Spectrom. 2015, 30, 254-259.

(26) Becker, J. S.; Zoriy, M. V.; Pickhardt, C.; Palomero-Gallagher, N.; K. Zilles K. Anal. Chem. 2005, 77, 3208-3216.

(27) Lu, H.; Hunt D. M.; Ganti, R.; Davis, A.; Dutt, K.; Alam, J.; Hunt, R.C. Exp. Eye Res. 2002, 74, 83-92.

(28) Nakamura, S.; Shimazawa, M.; Inoue, Y.; Takata, S.; Ito, Y.; Tsuruma, K.; Ikeda T.; Honda A.; Satoh M.; Hara, H. Invest. Ophthalmol. Visual Sci. 2014, 55, 68516860 .

(29) Nakazato, K.; Tomioka, S.; Nakajima K.; Saito H.; Kato M.; Kodaira T.; Yatsuzuka S.; Shimomura Y.; Hiroki T.; Motoyama K.; Kodama H.; Nagamine T. J. Trace Elem. Med. Biol. 2014, 28, 441-447.

(30) Milnerowicz, H.; Bizon, A. Acta Biochim. Pol. 2010, 57, 99-104. 
Table 1. Molecular mass obtained from the heavy and light chain of the Ab with and without bioconjugate with the AuNCs label. The molecular mass was determinate by the relative mobility of the different protein bands. Uncertainties represent the standard deviations of the mean of 3 independent measurements ( 3 independent gels).

\begin{tabular}{llc}
\hline & Chain & Molecular Mass (kDa) \\
\hline Antibody & Heavy & $62.36 \pm 5.9$ \\
\hline & Light & $27.19 \pm 2.1$ \\
\hline Ab-AuNCs Bioconjugate & Heavy & $72.62 \pm 7.3$ \\
\hline & Light & $29.65 \pm 2.8$ \\
\hline
\end{tabular}

Table 2. MT 1/2 concentration (expressed as ng protein per $\mathrm{g}$ of tissue) in the human retina obtained, by the ELISA kit, from tissue homogenates of different donors. Terms "RE" and "LE" correspond to "Right Eye" and "Left Eye" of the same donor. Uncertainties represent the standard deviations of the mean of 3 independent measurements.

\begin{tabular}{lccc} 
Donor & \multicolumn{3}{c}{ [MT 1/2] (ng g $\left.^{-1}\right)$} \\
\hline \#11203 & 89.12 & \pm & 10.04 \\
\hline \#11220 & 267.73 & \pm & 8.13 \\
\hline \#11221 & 18.82 & \pm & 3.01 \\
\hline \#11228 & 99.56 & \pm & 8.95 \\
\hline \#11232 RE & 145.49 & \pm & 11.56 \\
\hline \#11232 LE & 215.15 & \pm & 35.91 \\
\hline \#11242 RE & 124.37 & \pm & 2.84 \\
\hline \#11242 LE & 53.05 & \pm & 7.78 \\
\hline \#11253 RE & 84.07 & \pm & 13.81 \\
\hline \#11253 LE & 124.71 & \pm & 14.39 \\
\hline \#11278 & 79.13 & \pm & 13.98 \\
\hline
\end{tabular}


Figure 1. Optical image of the gel obtained after electrophoretic separation (Coomassie brilliant blue stain was used to identify the different protein bands). The lanes contain proteins from the following sources (left to right): A) Molecular weight size marker, B) AuNCs (without bioconjugation), C) Ab-AuNCs bioconjugate, and D) Ab (without bioconjugation).
(A)
(B)
(C)
(D)

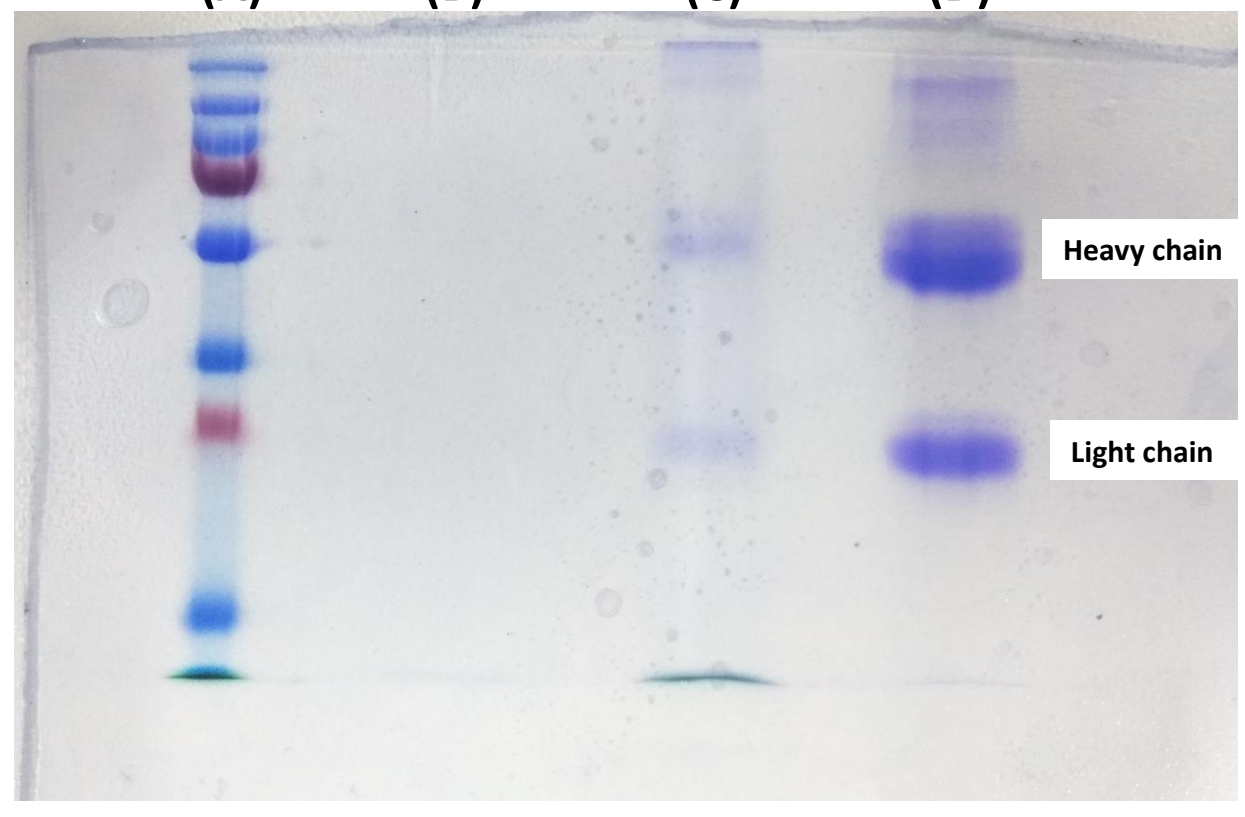


Figure 2. General diagram with the different steps required to transform $\mathrm{Au}$ concentration (from AuNC label used for bioconjugation) in analyte concentration into the tissue sections. Direction of the diagram is from left to right.

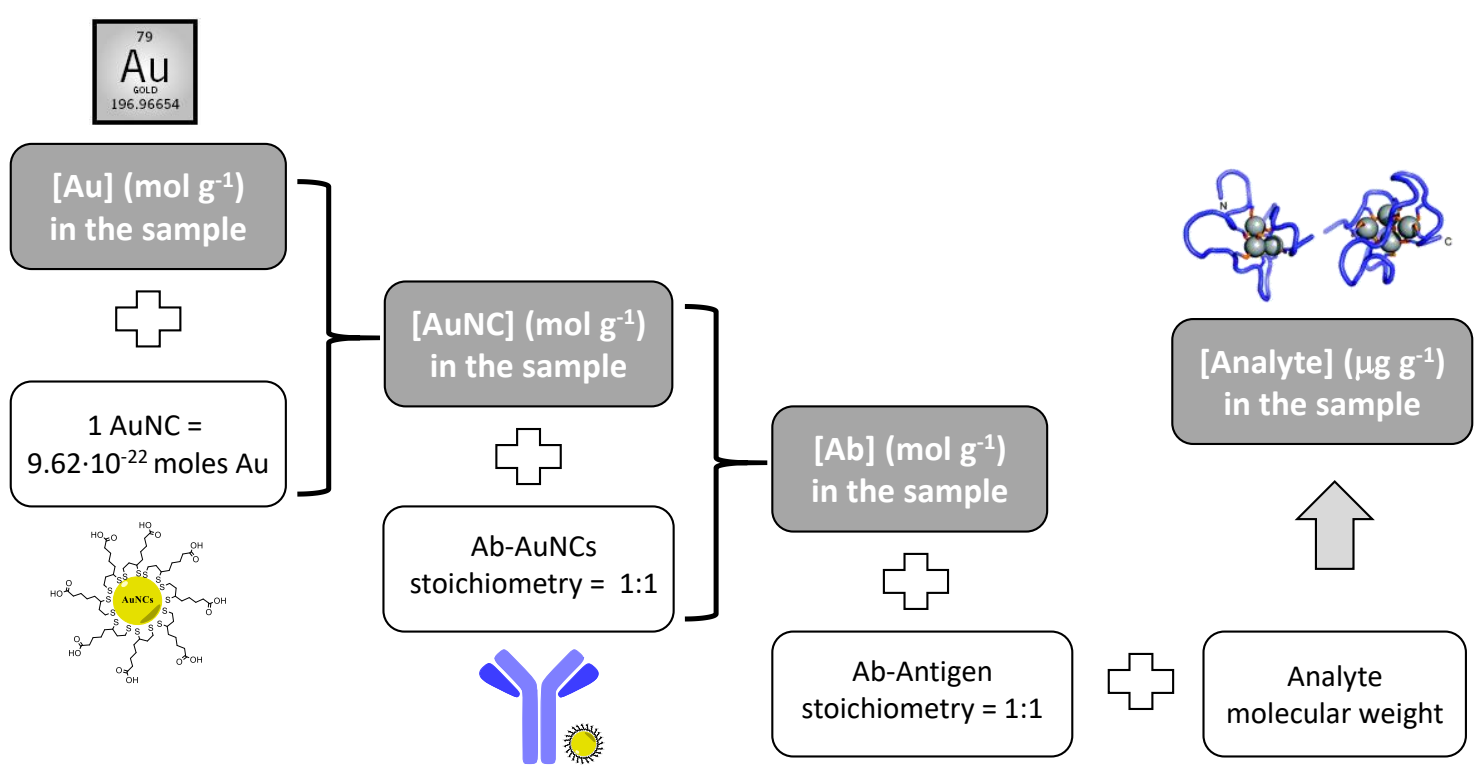


Figure 3. Quantitative images obtained for MT $1 / 2$ distribution in the human retina by LA-ICP-MS. A) Transmission image for the analyzed area from donor \#1 (PR: Photoreceptors; ONL: Outer Nuclear Layer; OPL: Outer Plexiform Layer; INL: Inner Nuclear Layer; IPL: Inner Plexiform Layer; GC: Ganglion Cells; NFL: Nerve Fiber Layer), B) Donor \#1, C) Donor \#2, D) Donor \#3, and E) Donor \#4.
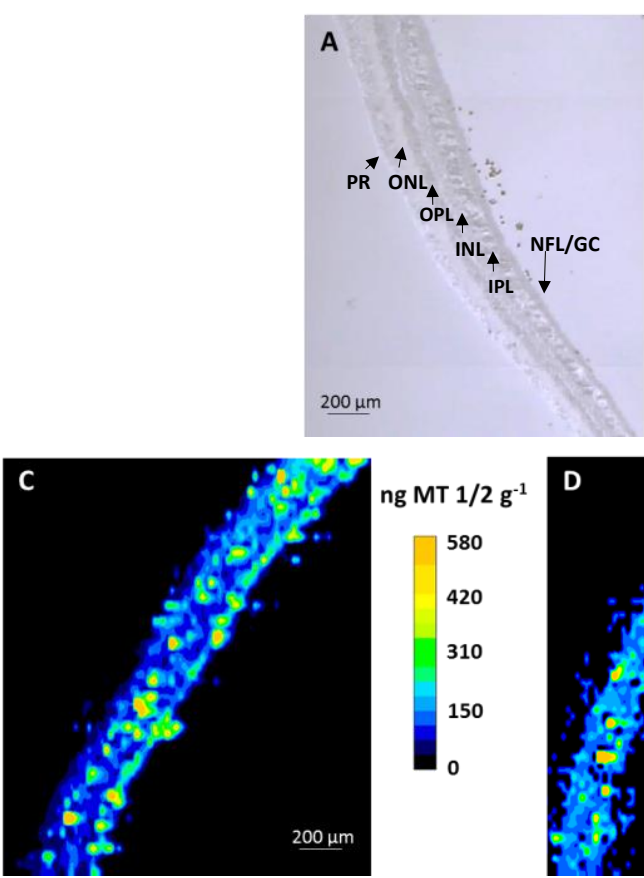
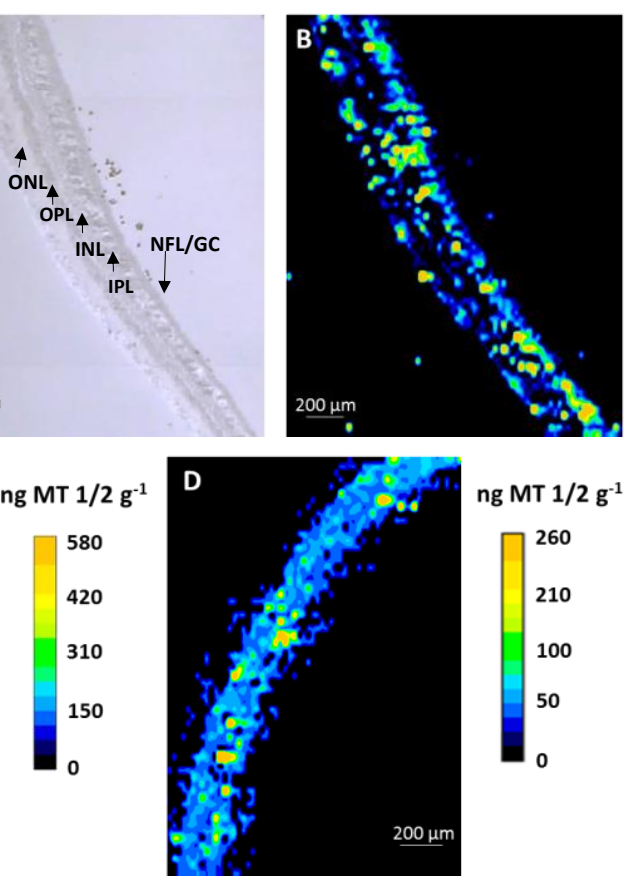

ng MT $1 / 2 \mathrm{~g}^{-1}$
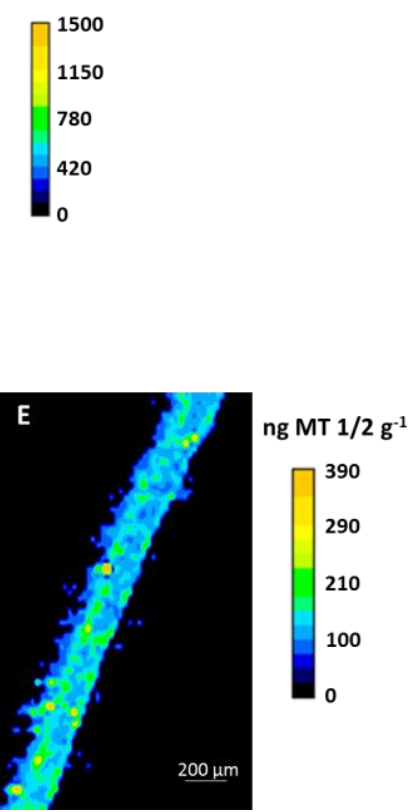


\section{Table of Contents Graphic}

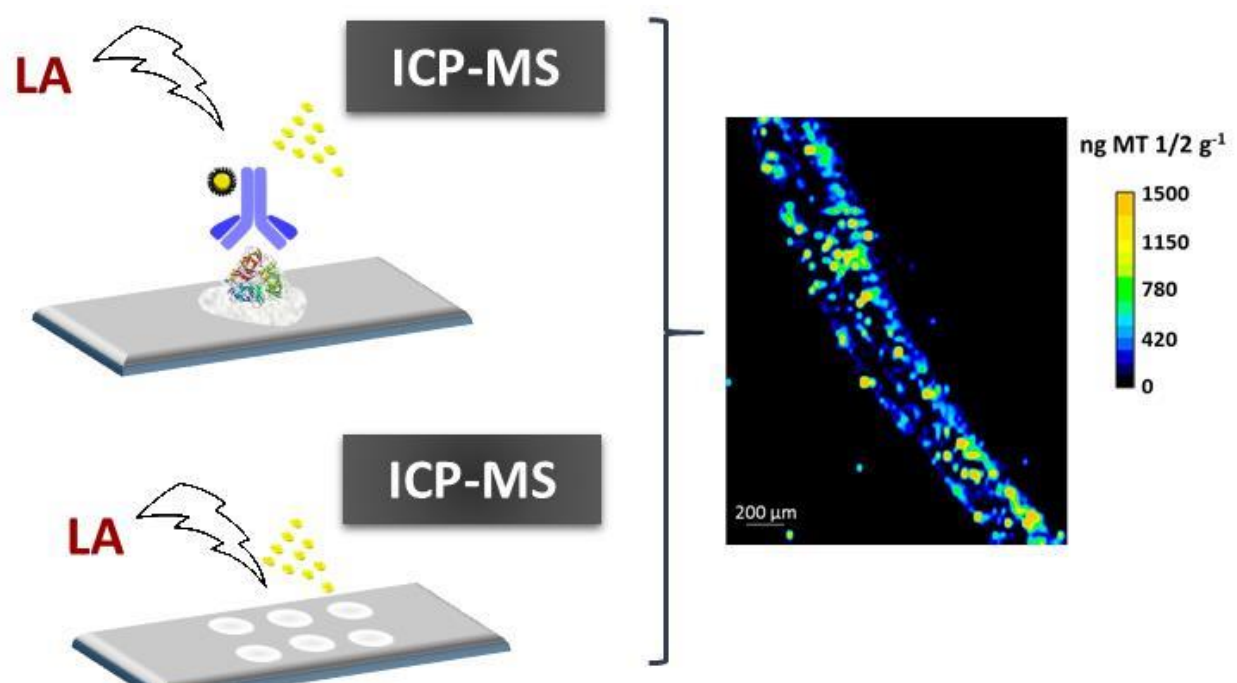

A sensitive methodology using antibody-conjugated AuNCs is developed for the absolute quantitative bioimaging of specific proteins in biological tissues by LA-ICP-MS 